\title{
Tagungen und Kongresse - Meetings and Conferences
}

\subsection{2.-13.02.1998 Bremen}

Deutschland

01.04.-09.04.1998 Bielefeld

Deutschland

30.04.-03.05.1998 Wien

Osterreich

21.05.-23.05.1998 Wien

Osterreich

06.10.-10.

10.

1998

Bern

Schweiz

10.10.-11.

10.

1998

Interlaken

Schweiz

19; 11.-22.11.1998 Düsseldorf

Deutschland

Rationale Konzepte der intensivmedizinischen Praxis -Gegenwart und Zukunft - 8.

Internationales Intensivmedizinisches Symposium

Transfusionsmedizinisches Seminar der DGTI für Ärzte im 2,/3. Weiterbildungsjahr zum

Facharzt für Transfusionsmedizin

2nd European Congress of Orthopedic and Trauma Anaesthesia

Trauma Care '98. 11. jährliches Symposium für Trauma-Anaesthesie und -Intensivmedizin 31. Jahrestagung der Deutschen Gesellschaft für Transfusionsmedizin und Immunhämatologie (DGTI)

Postkongreßsymposium «Extrakorporale Immunadsorption» 
MEDICA - Weltforum für Arztpraxis und Krankenhaus -28. Internationale Fachmesse und Kongreß

Auskunft: Prof. Dr. W. Kuckelt, Zentrum für Anästhesiologie, Klinik für operative und allgemeine Intensivmedizin, Zentralkrankenhaus links der Weser, Senator-Weßling-Straße 1, D28277 Bremen Tel: +49 421 879-731, Fax: -788

Auskunft: Prof. Dr. Reinhold Eckstein, Abt. Transfusionsmedizin und Hämostaseologie, Universitätsklinikum Erlangen/Nürnberg, Krankenhausstraße 12, D-91054 Erlangen Tel:+49 9131 856-46, Fax:-987

Auskunft: Alex M. Bleicher, APA 98, Postfach 45, A-1097 Wien E-mail apa98@akh-wien.ac.at Auskunft: ITACCS Austria, c/o Dr. Walter Mauritz, Postfach 185, A-1200 Wien

Fax:+43 3301976

Auskunft: Prof. Dr. Reinhold Eckstein, Abt. Transfusionsmedizin und Hämostaseologie, Universitätsklinikum Erlangen/Nürnberg, Krankenhausstraße 12, D-91054 Erlangen Tel.: +49 9131 856-46, Fax: -987

Auskunft: Prof. Dr. Reinhold Eckstein, Abt. Transfusionsmedizin und Hämostaseologie, Universitätsklinikum Erlangen/Nürnberg, Krankenhausstraße 12, D-91054 Erlangen Tel: +49 9131 856-46, Fax: -987

Auskunft: MEDICA Deutsche Gesellschaft zur Förderung der Medizinischen Diagnostik, Löffelstraße 1, D-70597 Stuttgart Tel: +49 71176-514 54, Fax: -69 92

\section{KARGER}

(C) 1997 S. Karger GmbH, Freiburg Fax (0761) 4520714 http://www.karger.ch 